A HIGH CURRENT, HIGH VOLTAGE SOLID-STATE PULSE GENERATOR FOR THE NIF PLASMA ELECTRODE POCKELS CELL*

P.A.Arnold, F.Barbosa, E.G. Cook, B.C. Hickman, G.L. Akana, C.A Brooksby

August 2, 2007

2007 IEEE International Pulsed Power and Plasma Science Conference albuquerque, NM, United States June 21, 2007 through June 22, 2007 
This document was prepared as an account of work sponsored by an agency of the United States Government. Neither the United States Government nor the University of California nor any of their employees, makes any warranty, express or implied, or assumes any legal liability or responsibility for the accuracy, completeness, or usefulness of any information, apparatus, product, or process disclosed, or represents that its use would not infringe privately owned rights. Reference herein to any specific commercial product, process, or service by trade name, trademark, manufacturer, or otherwise, does not necessarily constitute or imply its endorsement, recommendation, or favoring by the United States Government or the University of California. The views and opinions of authors expressed herein do not necessarily state or reflect those of the United States Government or the University of California, and shall not be used for advertising or product endorsement purposes.

This work was performed under the auspices of the U. S. Department of Energy by University of California, Lawrence Livermore National Laboratory under Contract W-7505-Eng-48. 


\title{
A HIGH CURRENT, HIGH VOLTAGE SOLID-STATE PULSE GENERATOR FOR THE NIF PLASMA ELECTRODE POCKELS CELL*
}

\author{
P.A. Arnold and F. Barbosa \\ National Ignition Facility, Lawrence Livermore National Laboratory, 7000 East Avenue \\ Livermore, CA, U.S.A. \\ E.G. Cook, B.C. Hickman and G.L. Akana \\ Beam Research Program, Lawrence Livermore National Laboratory, 7000 East Avenue \\ Livermore, CA, U.S.A. \\ C.A Brooksby \\ National Security Technologies, 161 South Vasco Road, Suite A \\ Livermore, CA, U.S.A.
}

\begin{abstract}
A high current, high voltage, all solid-state pulse modulator has been developed for use in the Plasma Electrode Pockels Cell (PEPC) subsystem in the National Ignition Facility. The MOSFET-switched pulse generator, designed to be a more capable plug-in replacement for the thyratron-switched units currently deployed in NIF, offers unprecedented capabilities including burst-mode operation, pulse width agility and a steady-state pulse repetition frequency exceeding $1 \mathrm{~Hz}$. Capable of delivering requisite fast risetime, $17 \mathrm{kV}$ flattop pulses into a $6 \Omega$ load, the pulser employs a modular architecture characteristic of the inductive adder technology, pioneered at LLNL for use in acceleration applications ${ }^{1}$, which keeps primary voltages low (and well within the capabilities of existing FET technology), reduces fabrication costs and is amenable to rapid assembly and quick field repairs.
\end{abstract}

[1]. E.G. Cook, et al., Solid State Modulator R\&D at LLNL, $1^{\text {st }}$ International Workshop on Recent Progress in Induction Accelerators, Tsukuba, Japan, Oct.2002.

\section{INTRODUCTION}

The addition of Advanced Radiographic Capability, i.e., short duration probe beams, to the National Ignition Facility of the Lawrence Livermore National Laboratory requires a new generation of switch pulse generator for the Plasma Electrode Pockels Cell. As currently deployed, PEPC interacts with the laser on the second and third passes through the laser's main amplifier, rotating the polarization of the light by two increments of $90^{\circ}$ by employing a single, high voltage pulse. On the other hand, ARC PEPC will be required to turn on for a second time approximately $1 \mu$ s later to interact with an energetic reflected beam, sending it into a beam dump, thus protecting the relatively low-power front end of the laser.

\section{BACKGROUND}

Described in detail in previous publications, the NIF PEPC is an enabling technology, permitting the facility to take advantage of a four-pass architecture, reducing the overall size and cost of the laser. ${ }^{1,2}$ Briefly, the Pockels cell, used in combination with an optical polarizer, forms an optical switch that can be employed to trap and then release a beam from an optical cavity, thereby taking full advantage of the available optical gain. PEPC interacts with the beam on its second and third passes through the main amplifier, introducing successive $90^{\circ}$ polarization rotations that allow the beam to pass through and then reflect off a thin film polarizer.

A thyratron-switched $6.25 \Omega$ pulse forming line has served as the basis for the switch pulse generator (SPG) that is currently deployed in large numbers in the facility. ${ }^{3}$ This pulse generator delivers a excitation that results in a 300ns (FWHM), $17 \mathrm{kV}$, flattop pulse being applied to a nonlinear crystal to produce the required birefringence. Transient helium plasmas serve as electrodes for coupling to the crystal. From an electrical standpoint the KDP crystal, sandwiched between two plasma electrodes looks like a capacitance of $\sim 6 \mathrm{nF}$. In the standard PEPC, this impedance is shunted by a series combination of a $7.5 \Omega$ resistor and a $75 \mathrm{nH}$ inductor; these elements were designed for the original PEPC to modify the rise- and falltimes of the applied pulse (offsetting to a degree the effects of the very large capacitance) and to give a slight voltage "step-up." The pulser is temporally isolated from

\footnotetext{
* This work performed under the auspices of the U.S. Department of Energy by the University of California Lawrence Livermore National Laboratory under Contract No. W-7405-Eng-48 and supported by the Laboratory for Laser Energetics under the U.S. Department of Energy - University of Rochester Cooperative Agreement No. DE-FC09-92SF19460.
} 
the load by $60 \mathrm{~m}$ of cable to minimize interaction and non-ideal transients.

Adding the probe beams of the ARC system places significant new requirements on PEPC. The added requirements may be understood in the following context: Energetic reflections, an inherent aspect of employing short duration, high power laser pulses, pose a threat to the low power "front end" of the laser. While the details of the optical beamline are beyond the scope of this discussion, the most straightforward solution for protecting the front end of the laser is to have PEPC turn on for a second time, approximately a microsecond after the laser gain pass through the main amplifier, rotate the polarization of the light by a third increment of $90^{\circ}$ and allow the light to be safely trapped in a beam dump. The concept is illustrated in the timing diagram of Figure 1.

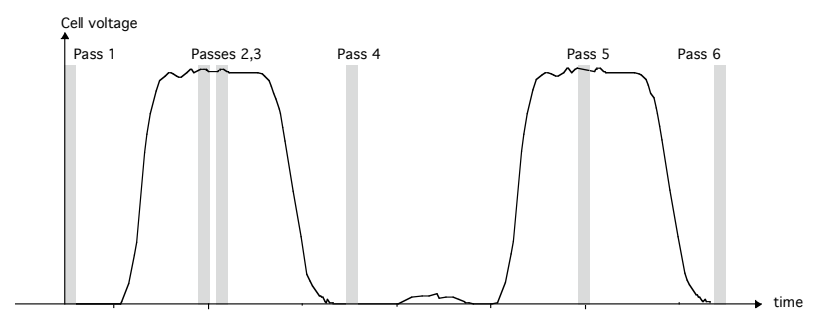

Figure 1. Representation of relative timing of optical pulses and cell voltage for ARC PEPC. Total time from Pass 1 to Pass 6 is $\sim 1.5$ microseconds. Passes $1-4$ represent standard PEPC performance.

While it appeared to be possible to adapt the existing technology to supply a second pulse, the chosen (and superior) path employs a topology that has been developed over the past several years by and for the LLNL Beam Research Program. ${ }^{4.5}$

\section{DISCUSSION}

The architecture of choice for the double-pulse SPG is a MOSFET-switched inductive adder. ${ }^{5}$ This circuit topology offers many features that are particularly wellsuited to the task at hand: the ability to generate flattop pulses with little droop, burst mode capability, continuously variable pulse separation/burst frequency agility, variable pulse width, as well as fast rise and fall times. Other features include the following:

- Modular design

- All drive components are ground referenced thus eliminating the need for floating/isolated supplies

- No high voltage grading is required

- Inherently low source impedance

- Pulse format defined by a programmable, low voltage pulse generator.

With reference to the modulator block diagram in Figure 2, the pulse generator employs multiple, stacked 1:1 transformers in which each transformer primary is separately excited. Secondary windings are connected in series so that the modulator output in sum of the primary voltages. (As implemented, the secondary is a continuous rod traversing all primaries.) Modular in nature and welladapted to printed circuit card implementation, the modulator employs a total of twenty-seven identical 750 $\mathrm{V}$ stages to achieve the required $17 \mathrm{kV}$ output. Each stage comprises twenty-four pairs of high speed MOSFETs in parallel (split between two identical circuit boards), and configured so that each pair of FETs has its own gate drive circuitry and storage capacitance. Boards plug directly into the housing of the transformer core. MOSFET switches are rated at $1200 \mathrm{~V}$ and are capable of conducting in excess of $80 \mathrm{~A}$ in pulsed mode. However, by operating with a peak current of only $\sim 62 \mathrm{~A}$ it is possible to significantly reduce the voltage drop across each device. The pulser is able to reliably deliver the nearly $3 \mathrm{kA}$ pulse required to provide a $17 \mathrm{kV}$ excitation at the Pockels cell. The risetime of the output pulse is approximately $20 \mathrm{~ns}$ while the falltime is purposely held to $\geq 20 \mathrm{~ns}$ to avoid excessive voltage transients in the system. These rise-and falltime parameters are achievable largely because of small inductance in the FET/capacitor/transformer primary loop. 


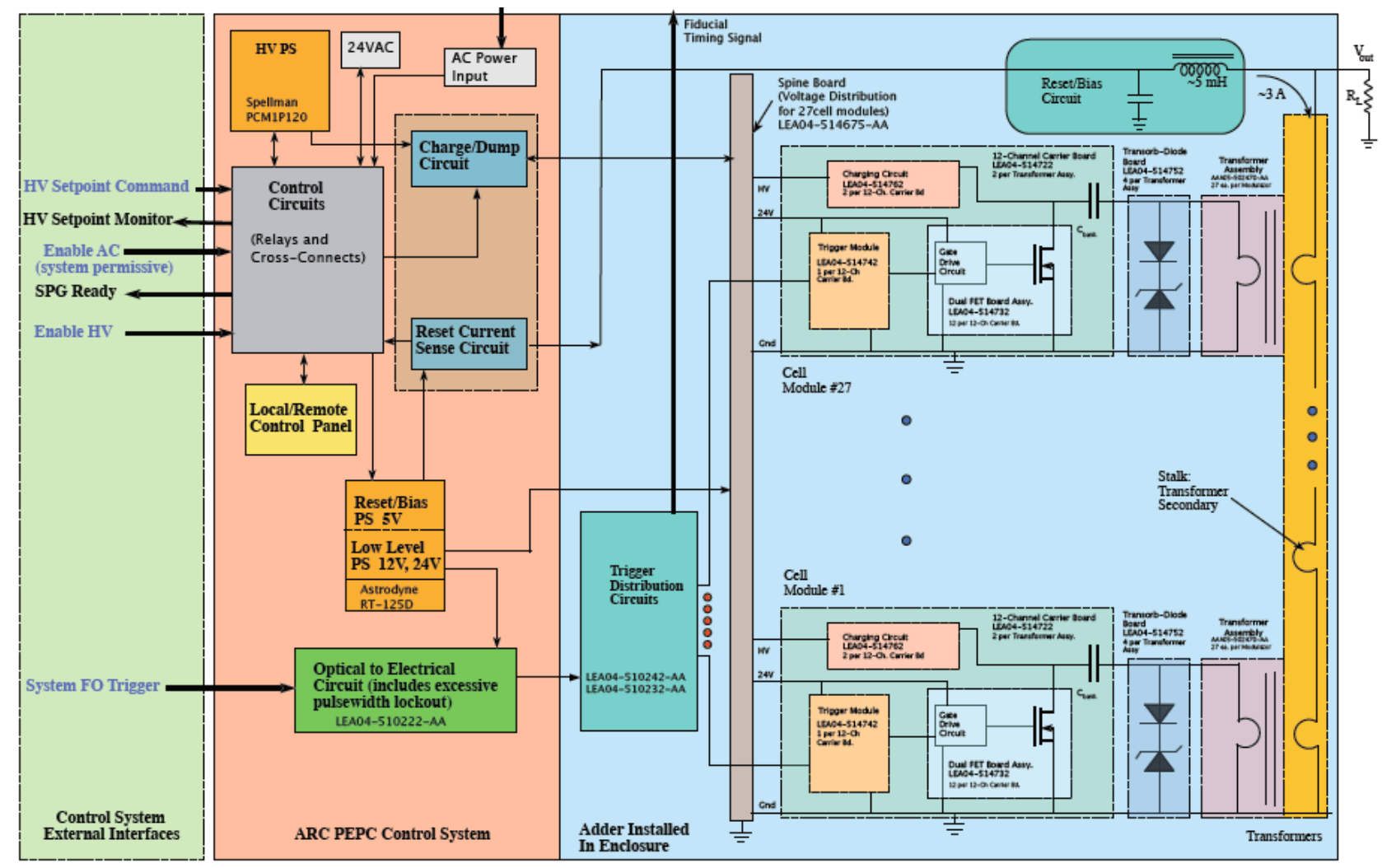

Figure 2. Block Diagram of ARC PEPC modulator.

Transient protection circuitry resides on separate boards that plug directly into the housing of the transformer core to minimize loop inductance. The high speed diodes allow currents established in the transformer to continue to flow while the MOSFETs turn off. A total of 20 diodes, providing a surge current rating of more than 10 $\mathrm{kA}$, is installed on each core. The large number of diodes, which at first blush seems excessive, is required to limit the diode forward voltage and properly protect the MOSFETs during the diode turn-on transient. Transorbs, also mounted on the same board, ensure that the reset current can develop sufficient voltage across the primary winding to properly reset the core. (Without the transorbs the voltage would be limited to the drop across the forward-biased diodes.)

Reset for the magnetic cores of each transformer is provided by an inductively isolated $5 \mathrm{~V} \mathrm{DC}$ power supply delivering approximately $2.5 \mathrm{~A}$ to the common single-turn secondary. Cores are wound with 0.8 mil Metglas ${ }^{\mathrm{TM}} 2605$ SA1, insulated turn-to-turn with Namlite. ${ }^{\mathrm{TM}}$ With reset the cores have an available $\Delta \mathrm{B}$ of $\sim 3 \mathrm{~T}$. The modulator is able to deliver a single pulse with a duration of $1.5 \mu$ s at full voltage without saturating the cores and/or exceeding the allowable droop. While the cores reset in the 5 seconds elapsing between bursts, no appreciable resetting can occur between pulses in the burst. To add an additional measure of protection against core saturation and its attendant problems, an passive integrator limits the total duration of the pair of pulses in a burst.

For temporal precision and noise immunity, the master trigger for the system is a single optical pulse. This pulse is then used to trigger a programmable trigger generator that in turn produces a pair of pulses that determine the duration and separation of the output pulses.

A single ended DC power supply provides the energy that is stored in the more than 400 on-board film capacitors. The input setpoint voltage of the supply is clamped to prevent overcharging of the system. Distribution of the supply voltage is the role of the spine board that spans the entire stack of modules.

The modulator has been designed with fault tolerance and maintainability in mind. In particular, the parallel nature of the system allows a number of components to fail before the modulator must be serviced. When service is required, LEDs incorporated into the boards guide the technician to the region, if not the specific part that requires maintenance. Virtually all components can be replaced by simply replacing the board on which they reside.

A fully operational modulator is depicted in Figure 3 with special emphasis on the semicircular 12-channel carrier board, which plugs into the housing of the core, making a reliable current connection via Ball-Spring. ${ }^{\mathrm{TM}}$ The pulser is housed in a standard 24" 336 " rack approximately 5' tall. Eight RG-217 coaxial cables plug 
into the top of the pulser and deliver the pair of high voltage pulses to the Pockels cell located a significant distance from the pulsers.

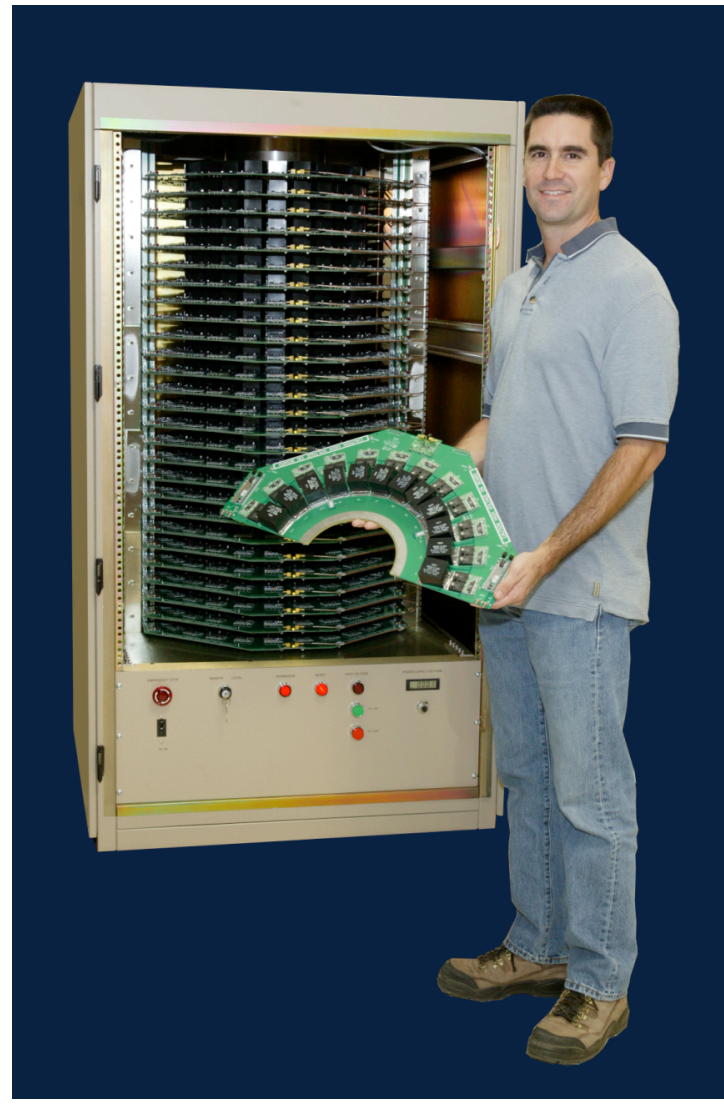

Figure 3. Operational Modulator, 27 modules high. The semicircular 12-channel carrier board plugs into transformer core housing using Ball-Spring approach.

\section{TEST RESULTS}

Four modulators have been operated successfully into both dummy loads and an operating plasma electrode Pockels cell, sustaining $1 \mathrm{~Hz}$ operation for days at a time and accumulating thousands of pulses. (Note that deployed modulators will run at $0.2 \mathrm{~Hz}$ for approximately 5 minutes to support each NIF shot.) A two-pulse burst from the modulator is shown in Figure 4. Note that the rise- and falltimes at the Pockels cell are heavily influenced by the capacitance of the crystal. As described previously, internal risetimes are on the order of $20 \mathrm{~ns}$ while cell rise- and falltimes are on the order of $100 \mathrm{~ns}$. Also of note are the inter-pulse interactions between the modulator and the cell. The majority of the activity is due to rise- and fall-generated reflections propagating back and forth between the cell and the modulator. While this level of distortion in the flattop of the second pulse is acceptable (given the sine-squared voltage dependence of a Pockels cell and the ability to adjust timing to offset the effects of interaction) there is an on-going effort to optimize/reduce cell reflections and interactions without having to resort to excessively long cables between the modulator and PEPC.

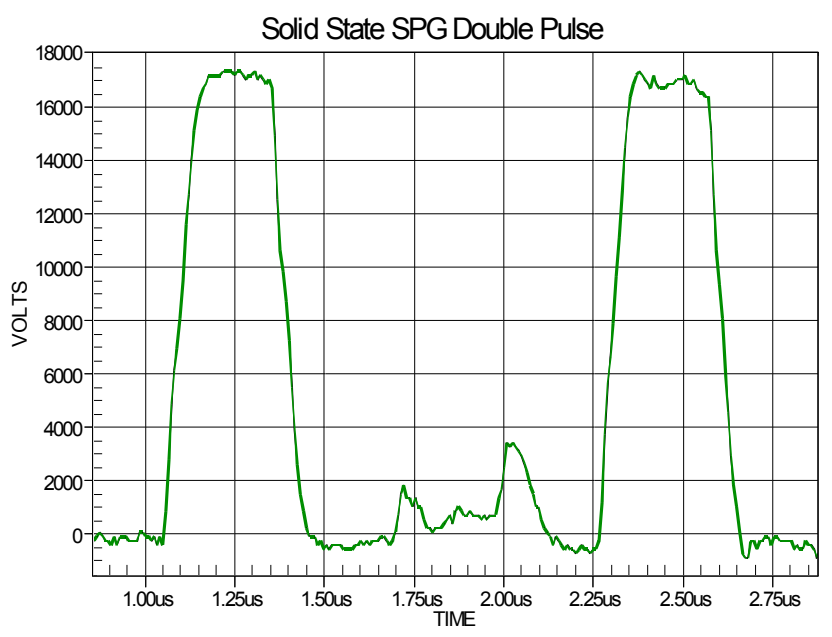

Figure 4. Double pulse applied to Plasma Electrode Pockels Cell. Rise- and falltimes and heavily dominated by capacitance of the load. Rising and falling edgegenerated reflections are obvious between pulses.

Figure 4 displays nominal pulsewidth of $\sim 300$ ns (FWHM), based on original PEPC requirements that were dictated by a fixed pulsewidth. However, pulsewidth agility is inherent in the new modulator design and can be utilized to accommodate probe beams that lead or lag the main laser pulses by significant amounts. The control system being developed and tested for ARC PEPC will set both pulsewidth and pulse separation in accordance with specific experimental requirements.

\section{CONCLUSION}

Inductor adder technology has been applied to the NIF ARC PEPC. Preliminary testing has shown the modulators to be reliable and fully capable of delivering the pair of required $17 \mathrm{kV}$ pulse to the cell with required reliability. Pulsewidth agility and burst capability are keys to the success of the modulator design. Development of the control system is on-going and deployment of the new system in NIF is expected within the calendar year. 


\section{REFERENCES}

[1] P.A. Arnold, C.W. Ollis, A.F. Hinz, C. Robb, K.A. Primdahl, J.J.Watson, M.D. O'Brien, W.G. Funkhouser, P.J. Biltoft, R.T. Shelton, W.C. Tapley and W.J. DeHope, Deployment, commissioning and operation of plasma electrode Pockels cells in the National Ignition Facility, SPIE Vol. 5341, Optical Engineering at the Lawrence Livermore National Laboratory II: The National Ignition Facility, pp. 156-167.

[2] P.A. Arnold, C.W. Ollis, A.F. Hinz, F. Barbosa, E.S. Fulkerson, Pulsed Power Aspects of the NIF Plasma Electrode Pockels Cell, Proceedings of the $15^{\text {th }}$ IEEE Pulsed Power Conference, Monterey CA 2005.

[3] F. Barbosa, P.A, Arnold, A.F. Hinz, C.W. Ollis, R.A. Zacharias, E.S. Fulkerson, C.L. Bishop, Plasma Electrode Pockels Cell Subsystem Performance in the National Ignition Facility, Proceedings of the $16^{\text {th }}$ IEEE Pulsed Power Conf, Albuquerque NM, 2007.

[4] H.C. Kirby, et al., Development of Solid-state Induction Modulators for High PRF Accelerators, Proceedings of the $10^{\text {th }}$ IEEE Pulsed Power Conference, Albuquerque NM, 1995.

[5] E.G. Cook, et al., "Solid State Modulator R\&D at LLNL," $1{ }^{\text {st }}$ International Workshop on Recent Progress in Induction Accelerators, Tsukuba, Japan, Oct. 2002. 